REGARDS

SUR LEECONOMIE ALLEMANDE

BULLETIN ECONOMIQUE DU CRAC

\section{Regards sur l'économie allemande}

Bulletin économique du CIRAC

$83 \mid 2007$

Varia

\title{
Conseil économique : un nouveau groupe d'instituts
}

\section{Isabelle Bourgeois}

\section{(2) OpenEdition}

1 Journals

Édition électronique

URL : http://journals.openedition.org/rea/602

DOI : $10.4000 /$ rea.602

ISBN : 978-2-8218-0862-1

ISSN : 1965-0787

\section{Éditeur}

CIRAC

Édition imprimée

Date de publication : 1 octobre 2007

Pagination : 37-38

ISSN : 1156-8992

Référence électronique

Isabelle Bourgeois, «Conseil économique : un nouveau groupe d'instituts », Regards sur l'économie allemande [En ligne], 83 | octobre 2007, document 2, mis en ligne le 01 octobre 2009, consulté le 15 septembre 2020. URL : http://journals.openedition.org/rea/602

Ce document a été généré automatiquement le 15 septembre 2020.

(c) CIRAC 


\title{
Conseil économique : un nouveau groupe d'instituts
}

\author{
Isabelle Bourgeois
}

1 A l'issue d'une procédure européenne d'appel d'offre, le gouvernement fédéral a retenu le 3 juillet un nouveau groupe de huit instituts de recherche économique indépendants chargés dans les trois années à venir de la réalisation des rapports conjoints de printemps et d'automne sur l'évolution et les perspectives de l'économie allemande. Dorénavant, ces experts auront également pour mission de proposer au gouvernement des orientations d'action pour sa politique économique et sociale.

2 Les huit organisations retenues sont:

- l'institut ifo de Munich et son partenaire, le centre de recherche économique Konjunkturforschungsstelle de l'Université Technique de Zurich,

- l'IfW auprès de l'Université de Kiel ;

- l'IWH de Halle et ses partenaires : l'Institut für Makroökonomie und Konjunkturforschung (IMK) de Düsseldorf et l'Österreichisches Institut für Wirtschaftsforschung (Vienne),

- ainsi que l'institut RWI (Essen), en partenariat avec l'Institut für Höhere Studien (Vienne).

3 Quatre de ces institutions (ifo, IfW, IWWH et RWI) appartenaient au groupe des six grands instituts précédemment chargés de la réalisation deux fois par an des rapports conjoncturels conjoints. La candidature des deux autres (le DIW de Berlin et le HWWA de Hambourg) n'a pas été retenue. Le choix d'un nouveau groupe d'experts indépendants, présenté comme "un potentiel d'innovation prometteur " par le ministère fédéral de l'Economie, s'inscrit aussi dans le prolongement d'un décalage croissant observé depuis plusieurs années entre la logique d'expertise macro-économique et la logique de décision politique (voir REA 78/06) en Allemagne.

Quelles qu'aient été les raisons internes de cette décision, la non reconduction des instituts DIW et HWWA est révélatrice de cette tendance, corollaire d'une autre: la difficulté croissante à concilier approche scientifique pure (avec ses critères propres) et approche opérationnelle, axée sur le conseil et le débat dans l'espace public. L'institut HWWA de Hambourg avait ainsi été sorti dès 2006 de la liste des instituts bénéficiant 
des subventions publiques versées par la Wissensgemeinschaft Gottfried Wilhelm Leibniz (WGL) sur la base de leur production scientifique. Or les critères purement quantitatifs de l'évaluation (une publication par collaborateur et par an dans une revue de réputation internationale) avaient accru pour cet institut le dilemme inhérent à la double activité de recherche et de conseil politique, l'incitant à se recentrer sur la recherche économique pure. Quant au cas du DIW, il est d'une certaine manière plus politique en ce sens qu'il reflète une réactivation des mécanismes institutionnels de ce 'corporatisme' qui est à la base de l'organisation des processus de prise de décision outre-Rhin. Depuis longtemps, l'approche néo-keynesienne de cet institut avait mauvaise image, ou plutôt: celle de son directeur du département des analyses conjoncturelles, Gustav Horn. Ce dernier avait donc fini par quitter le DIW en 2004, pour créer auprès de la Fondation Hans-Böckler (qui est entre autres le centre de recherche de la confédération syndicale DGB) un nouvel institut: l'Institut für Makroökonomie und Konjunkturforschung (IMK). Si cette 'scission' a forcé le DIW à recentrer ses activités sur la recherche tout comme le HWWA, le choix de l'IMK comme membre du nouveau groupe d'experts montre que le gouvernement de coalition est loin de s'opposer farouchement à une doctrine économique fondée sur la demande mais à la condition qu'elle soit formulée par un organisme qu'on puisse identifier comme l'émanation du mouvement syndical.

\section{INDEX}

Mots-clés : conjoncture, conseil économique, institut de recherche 